(C) IEEE. Personal use of this material is permitted. However, permission to reprint/republish this material for advertising or promotional purposes or for creating new collective works for resale or redistribution to servers or lists, or to reuse any copyrighted component of this work in other works must be obtained from the IEEE.

This material is presented to ensure timely dissemination of scholarly and technical work. Copyright and all rights therein are retained by authors or by other copyright holders. All persons copying this information are expected to adhere to the terms and constraints invoked by each author's copyright. In most cases, these works may not be reposted without the explicit permission of the copyright holder. 


\title{
MULTIPLE RE-WATERMARKING USING VARYING WAVELET PACKETS
}

\author{
Jutta Hämmerle-Uhl, Michael Liedlgruber, Andreas Uhl, and Hartmut Wernisch \\ Department of Computer Sciences, University of Salzburg, Austria \\ Corresponding author E-mail: andreas.uhl@ sbg.ac.at
}

\begin{abstract}
Wavelet packets are shown to be a possible means to limit watermark interference in multiple re-watermarking algorithms. It turns out that when demanding a certain distance among wavelet packet subband trees, a significant improvement of watermark detection correlation values may be achieved in such a scenario.
\end{abstract}

Index Terms - multiple watermarking, re-watermarking, robust watermarks, wavelet packets

\section{INTRODUCTION}

Watermarking has been proposed as a generic technique to solve various problems associated with topics in the areas of digital rights management (DRM) and multimedia security. Whereas watermarking has evolved to a mature technology in the last decade, several issues remain to be solved until large scale deployment is to be expected. Multiple watermarking is one of those issues.

Multiple watermarks can be used to address multiple applications or one application may be addressed several times [1]. Focusing on the way how single watermarking techniques are actually fused into multiple watermarking schemes, Sheppard et al. [2] distinguish three main categories of multiple watermarking techniques:

1. Composite watermarking: All watermarks are combined into a single watermark which is subsequently embedded in one single embedding step.

2. Segmented watermarking: The host data is partitioned into disjoint segments a priory and each watermark is embedded into its specific share.

3. Successive watermarking: Watermarks are embedded one after the other. This approach is also denoted Re-watermarking in literature.

In this work, we focus on multiple fingerprinting using rewatermarking employing robust embedding techniques. A condensed overview of multiple watermarking technology as described in literature is given in Section 2 in addition to a description of technological requirements for our target application scenario. Section 3 , being the main contribution of this work, introduces a wavelet packet based concept for multiple watermarking enforcing a certain amount of disjointness in frequency space of the wavelet packet subband structures employed. Experiments show improvements over the standard technique using a fixed pyramidal decomposition. Section 4 concludes the paper.

The work described in this paper has been partially supported by the Austrian Science Fund, project no. 19159.

\section{MULTIPLE RE-WATERMARKING}

Most of the research effort in the field of multiple watermarking has been invested into segmented watermarking, however, not a time or spatial domain segmentation is used but instead the data partitioning is employed in a transform domain in most cases (e.g. wavelet-based techniques have been described embedding multiple marks into different subbands - see e.g. [3]). There are differences concerning the purpose of multiple embedding of robust watermarks. Whereas the majority of the schemes developed aims at embedding a single watermark multiple times in order to increase the robustness, only few suggestions focus on embedding actually different watermarks (e.g. [2]). We aim at the latter scenario as described in the following.

The embedding of unique watermarks for receiver identification is called fingerprinting. In case a cover medium is sold, it may be of interest that information concerning both, the original owner and the recipient, are embedded. In case re-selling occurs, each time the cover medium is sold the corresponding informations can be embedded using watermarking technology. In this case we can trace back the way of the cover medium to its origin and are able to reconstruct the entire trading chain. We want to support this scenario with multiple watermarking technology. Fingerprinting solves the question what to embed but not how to embed it.

Composite watermarking is not very useful in this scenario since the multiple watermarks are embedded simultaneously. Segmented watermarking suffers from the fact that at least the approximate number of watermarks to be embedded needs to be known in advance to guarantee at least sub optimal usage of the available resources (e.g. embedding capacity). Therefore, successive or re-watermarking seems to be the most promising approach for our target scenario.

Fig. 1 visualizes the basic concept of our target scenario. We embed three marks $(A, B, C)$ successively into the image $I$ using some embedding technique $\oplus$. After inserting the marks, we result in the respectively marked images $I_{A}, I_{A, B}$, and $I_{A, B, C}$ from which the watermarks have to be extracted accordingly.

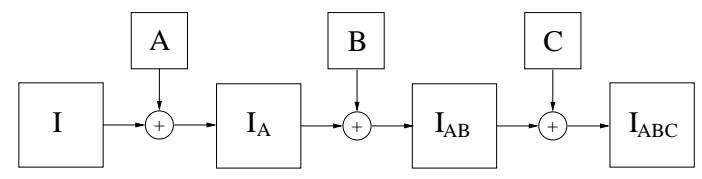

Fig. 1. Multiple Re-Watermarking scenario.

This scenario has been already investigated in literature. Two independent studies [2, 4] report on multiple re-watermarking by simply embedding different marks successively. Decreasing watermark correlations for an increasing number of embedded marks is found in the case of non-blind algorithms, whereas generally lower but not further decreasing correlation values are seen for blind algorithms. 
In this work, we propose a strategy how the observed watermark interference can be avoided or decreased. In a recent systematic investigation [5] we have shown that the use of different frequency bands for embedding is more efficient for avoiding watermark interference in re-watermarking as compared to the use of just different embedding domains. In the following, we exploit this observation by using varying wavelet packet decomposition structures for watermark embedding in multiple re-watermarking. On the one hand, the proposed approach is a sort of segmented watermarking, since the general idea of partitioning frequency space is employed. However, we do not demand a strictly disjoint partition and we do not require a priori knowledge about the number of watermarks to be embedded. Therefore, the suggested solution can be used for successive watermarking on the other hand.

\section{VARYING WAVELET PACKET SUBBAND STRUCTURES IN RE-WATERMARKING}

In previous work, we have employed key-dependent wavelet packet subband structures as a means to enhance security of the underlying watermarking schemes and could demonstrate increased resistance against unauthorized detection attacks and against unauthorized removal attacks [6] by using the idea of secret key-dependent embedding domains.

The strategy for the multiple embedding approach is as follows: we generate a wavelet packet subband structure randomly and insert the first watermark using this decomposition structure for embedding. Two variants for generating subband structures are used:

Decomposition 1 For this method we start at the root of the decomposition quadtree and use a 50\% probability for each subband in the tree to decide whether it should be decomposed further or not. This strategy potentially generates all possible decomposition trees.

Decomposition 2 Decomposition 1 tends to generate shallow quadtrees since the existence of a subband located at a high level in the tree requires several "decompose" decisions in a row. This has a significantly lower probability of course than the sum of probabilities of all the possible decisions leading to a non-existence of that subband. Therefore a technique from [6] is adopted to randomly generate subband structures where all possible trees are of almost equal occurrence probability.

For embedding the next watermark, we generate the next random subband structure, use it for embedding, and so on. This is repeated until all watermarks are embedded. However, randomly selecting frequency subbands for embedding is not much different to randomly distributing a watermark across different frequencies - basically, this approach does spread spectrum in a full wavelet packet decomposition, so there is not much reason why this technique should be better than pure spread spectrum in the pyramidal wavelet domain alone.

Therefore, in order to improve on pure spread spectrum, we introduce a measure to determine the distinctiveness of the generated quadtrees to gain control over the frequency bands used for embedding.

\subsection{A Quadtree Distance Measure}

A decomposition string is a string representation of a quadtree which is obtained by an pre-order traversal (i.e. depth-first traversal) of the quadtree. Let $D S$ be the decomposition string of a quadtree $T$, which is initialized as an empty string. Then, during the tree traversal, for each node a $1,2,3$ or 4 is appended to the string, if the first, the second, the third or the fourth child node is further subdivided, which means it contains more children nodes. Additionally an " $U$ " is appended to the string if there are no more subdivisions in the children nodes and the traversal goes back one level towards the root.

Fig. 2.b shows an example quadtree which results in the decomposition string

\section{UU32UUU2U312UU33UU4 3UUU 434 UU 44 UUU}

where the numbering scheme shown in Fig 2.a is used.

\begin{tabular}{|l|l|}
\hline 1 & lower left quadrant \\
2 & lower right quadrant \\
3 & upper left quadrant \\
4 & upper right quadrant \\
\hline
\end{tabular}

(a) Numbering

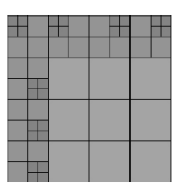

(b) Example

\begin{tabular}{|l|l|}
\hline B1 & 0.1 \\
B2 & 0.08 \\
B3 & 0.06 \\
B4 & 0.86 \\
\hline
\end{tabular}

(c) Distances
Fig. 2. Quadtree measure generation and example results.

Using this procedure the decomposition strings $D S_{1}$ and $D S_{2}$ for two given quadtrees $T_{1}$ and $T_{2}$ are created. The strings are compared character by character. As long as the strings are identical we know that the underlying quadtree structure is identical too. A difference at some position in the strings means that one of the strings has further child nodes where the other quadtree has no child nodes. When such a situation arises the decomposition string which contains more children nodes at this position is scanned further until the string traversal reaches again the quadtree position of the other quadtree node which has no more children nodes. During this process for each character of the first string the difference value $d v$ is updated according to the depth level of the different children nodes as follows: $d v=d v+\frac{1}{d}$, where $d$ is the depth of the children nodes not contained within the other quadtree. This process is repeated until $D S_{1}$ and $D S_{2}$ are scanned until their ends. $d v$ then represents the number of different nodes with the according depth levels taken into account. Therefore a difference in upper levels of the quadtrees contributes more to the difference value than a difference deeper in the quadtree. Finally, to norm the distance value between 0 and 1 the resulting difference value is divided by the sum of the lengths of $D S_{1}$ and $D S_{2}$.

Fig. 3 shows four quadtrees B1 - B4 which exhibit a different amount of similarity with tree A. Obviously, the tree most similar to A is B3, followed by B2 and B1. B4 is most dissimilar. The example values of our distance measure shown in Fig. 2.c exactly reflect this observation.

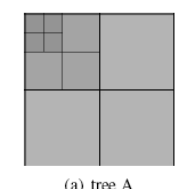

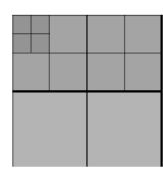

(b) tree B1

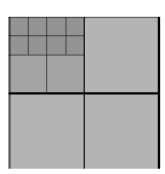

(c) tree $\mathrm{B} 2$

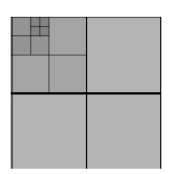

(d) tree B3

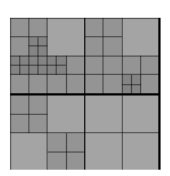

(e) tree B4
Fig. 3. Quadtree examples. 


\subsection{Experimental Setting and Results}

For illustrating our approach we have chosen the two well known 8 bpp and $512 \times 512$ pixels images "Lena" and "Barbara" for which we provide selected results. We have based our software on the "Watermarking Toolbox" ${ }^{1}$ developed by Peter Meerwald for watermark embedding and detection, unless denoted otherwise all embeddings have been done with the default parameters of the implementation. For demonstrating our approach, we employ the wavelet-based watermarking scheme of Wang et al. [7]. In this algorithm, a Gaussian distributed watermark is added in a specifically scaled way to the most significant wavelet coefficients, which are selected based on successive subband quantization (SSQ). This procedure originates from the Multi-Threshold Wavelet Codec (MTWC) developed by the same authors. We denote this algorithm as MTWWM (MultiThreshold Wavelet Watermarking) in the following.

Since the watermarking algorithm used is a non-blind technique, it is operated with correct reference images (images potentially already containing several marks before embedding the mark subject to detection, e.g. $I_{A}$ for detecting mark $B$ and $I_{A, B}$ for detecting mark $C$ - instead of the original $I$ ) in the detection process. All experiments have been repeated 50 times using randomly chosen watermarks, averaged correlation results are presented as well as the interval of all values observed. In these experiments, different watermarks and different embedding keys are chosen each time for embedding.

Fig. 4 illustrates the observations concerning watermark interference as described in the previous section.

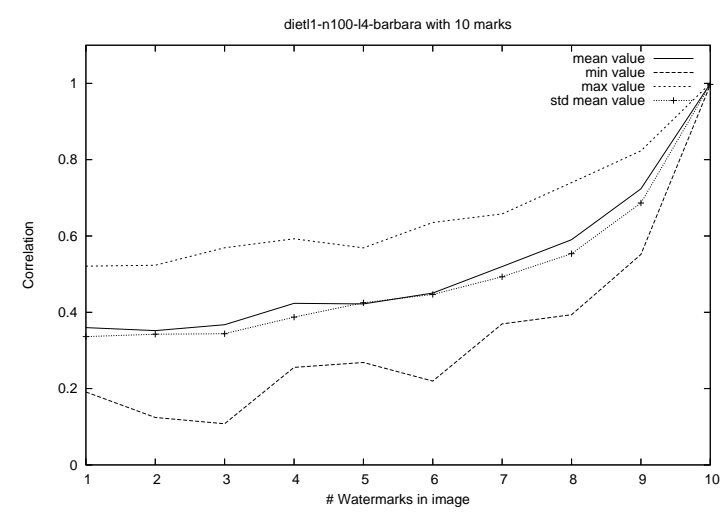

Fig. 4. Detection response of MTWWM algorithm (4 levels, length 100) with Decomposition 1 for embedding 10 watermarks, Barbara image.

The polygon denoted as std mean value shows the average detection correlation values resulting from re-watermarking using the standard algorithm with pyramidal wavelet decomposition. The result graphs (and all following graphs as well) have to be read and interpreted as follows: 10 watermarks have been embedded and the final image containing all marks is fed into the detection process for all marks. The mark at the rightmost position on the $\mathrm{x}$-axis has been embedded as the last mark, all other indicated positions on the $\mathrm{x}$-axis are ordered in time in the same manner. Note that the number on the $\mathrm{x}$-axis therefore does not denote the number of embedded marks (since it is always 10) but visualizes the time order in which the 10 marks have been embedded.

We notice a typical behaviour for non-blind algorithms when used in multiple re-watermarking: whereas the last embedded water-

\footnotetext{
${ }^{1}$ http: //www. cosy.sbg.ac.at/ pmeerw/Watermarking/
}

mark can be detected with a correlation value equal to that of single embedding, the detection correlation value decreases for watermarks further left in the plot (embedded at an earlier stage).

The decrease in correlation is obviously due to watermark interference which is strongest for the first mark embedded (at the leftmost position) since the signal extracted for detection (the difference between the marked image containing all embedded marks $I_{A, B, C}$ and the original image $I$ in this case) is a signal involving all embedded watermarks. In the MTWWM algorithm, large transform coefficients are selected to be manipulated for watermark embedding - on the one hand the set of marked coefficients changes from one marking stage to the next (which causes the detection to lose synchronization), on the other hand a significant number of coefficients is selected repeatedly, which causes the watermark embedding process to partially overwrite the previously embedded information which degrades correlation.

As a first step to improve detection correlation, we employ randomly chosen wavelet packet subband structures for embedding. Fig. 4 indeed clearly confirms our expectations that this strategy alone does not at all improve the detection correlation in our scenario - the polygon denoted as mean value shows corresponding results and exhibits almost identical values as those obtained with the standard (pyramidal) MTWWM technique. Identical results (i.e. no improvements) are observed for Decomposition 2 as well.

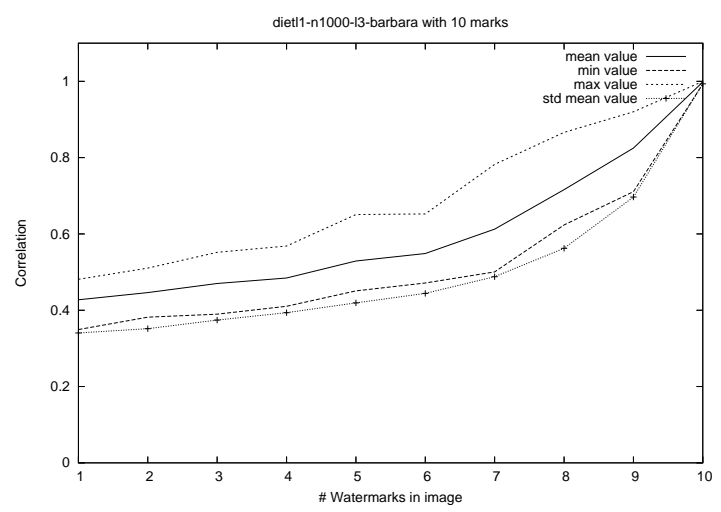

Fig. 5. Detection response of MTWWM algorithm (3 decomposition levels, length 1000) with Decomposition 1 for embedding 10 watermarks, tree distance enforced, Barbara image.

Using now the wavelet packet approach in combination with the quadtree distance measure, we randomly generate wavelet packet subband structures, but for embedding we accept only those generated trees, which exhibit a certain amount of dissimilarity as compared to all other trees used for watermark embedding before. For a distance value $>0.2$, we see that the correlation values are improved significantly (Figs. 5 to 7).

While for Decomposition 1 correlation is improved with different extent for the two images, average correlation for Decomposition 2 is improved consistently by 0.2 as compared to the standard case and even the lowest values found are slightly better then the average values of the standard algorithm. Obviously, the greater richness of the subband structures of Decomposition 2 leads to better results as compared to Decomposition 1 which suffers from the shallowness of its trees.

Thus, demanding a certain degree of tree difference makes sense since this obviously corresponds to a lower degree of overlap in frequency resolution. As a by-product, we could even improve image quality in some cases (e.g. by $2 \mathrm{~dB}$ PSNR for the Lena image). Sim- 


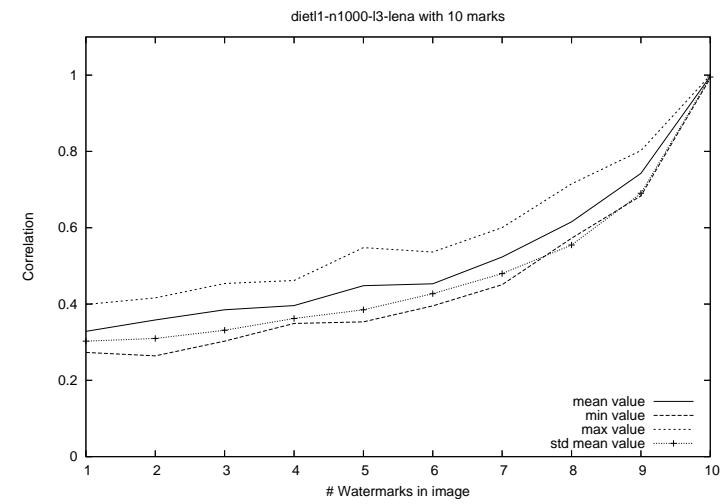

Fig. 6. Detection response of MTWWM algorithm (3 decomposition levels, length 1000) with Decomposition 1 for embedding 10 watermarks, tree distance enforced, Lena image.

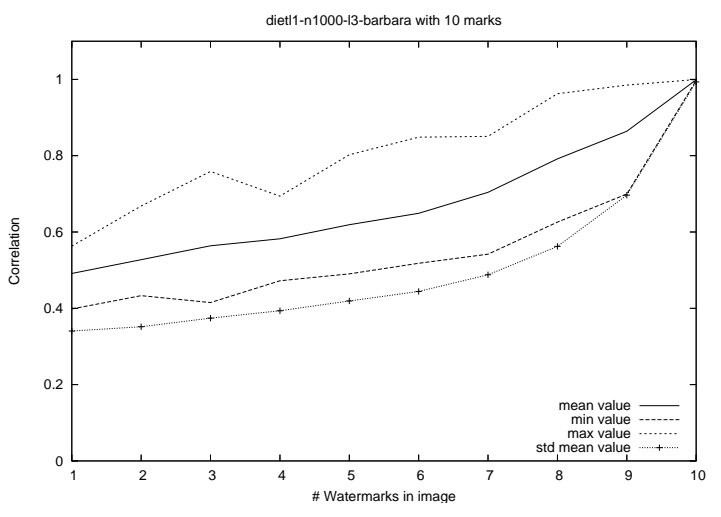

diet11-n1000-I3-lena with 10 marks

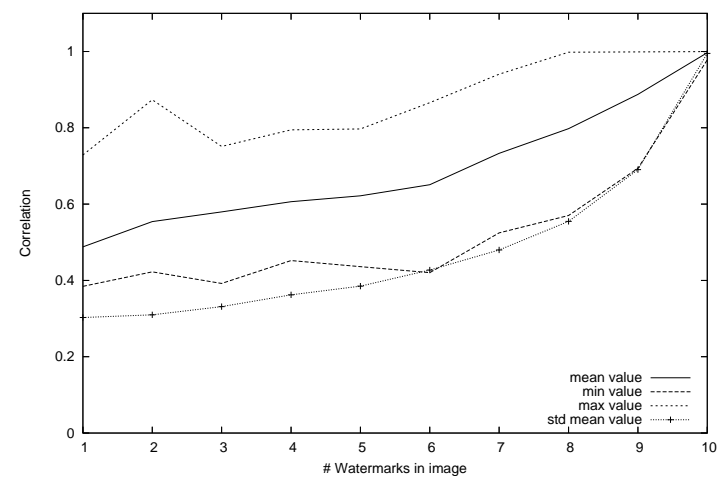

Fig. 7. Detection response of MTWWM algorithm (3 decomposition levels, watermark length 1000) with Decomposition 2, tree distance enforced, Barbara and Lena images.

ilar results with respect to detection correlation improvement and PSNR are obtained for a wide class of images.

In an actual application, the tree structures used for embedding marks in a given image could be considered part of the metainformation and can be stored in the format header of the file or in MPEG-7 descriptor fields in order to facilitate the next embedding stage to use a distinct structure again.

It should be noted however that the use of the proposed scheme is limited to cooperative environments where each embedding party is interested to maintain the detectability of the previously embedded watermarks and its own embedded mark. If this is not the case (i.e. a subband structure previously used for an "older" watermark is intentionally re-used for some purpose) the advantage of higher detection correlation is lost and the performance of the scheme reduces to that of the standard technique operating in the pyramidal wavelet domain. To maintain the advantage under such conditions, the subband structure information has to be encrypted.

\section{CONCLUSIONS AND FUTURE WORK}

It has been demonstrated that watermark interference in waveletbased multiple re-watermarking algorithms can be controlled in principle by using wavelet packet subband structures exhibiting a certain amount of dissimilarity. In future work we will address the following issues:

- Extension of the technique to blind and quantization-based watermarking schemes.

- Instead of randomly generating and assessing trees, a constructive solution should be found to generate the tree with the maximum distance to a set of given trees.

- Estimation of the number of trees available exhibiting a certain distance among each other.

\section{REFERENCES}

[1] F. Mintzer and G. W. Braudaway, "If one watermark is good, are more better?," in Proceedings of the International Conference on Accoustics, Speech, and Signal Processing, Phoenix, Arizona, USA, May. 1999, vol. 4, pp. 2067-2070.

[2] N. P. Sheppard, R. Shafavi-Naini, and P. Ogunbona, "On multiple watermarking," in Proceedings of the ACM Multimedia and Security Workshop 2001 (MMSW-01), Ottawa, Canada, Oct. 2001, pp. 3-6, ACM Press.

[3] C. S. Lu, S.-K. Huang, C. J. Sze, and H. Y. M. Liao, "Cocktail watermarking for digital image protection," IEEE Transactions on Multimedia, vol. 2, no. 4, pp. 209-224, Dec. 2000.

[4] A. Mascher-Kampfer, H. Stögner, and A. Uhl, "Multiple rewatermarking scenarios," in Proceedings of the 13th International Conference on Systems, Signals, and Image Processing (IWSSIP 2006), B. Enyedi and A. Reichardt, Eds., Budapest, Hungary, 2006, pp. 53-56.

[5] Daniel Mark, Andreas Uhl, and Hartmut Wernisch, "Experimental study on watermark interference in multiple rewatermarking," in Security, Steganography, and Watermarking of Multimedia Contents IX, Edward J. Delp and Ping W. Wong, Eds., San Jose, CA, USA, Jan. 2007, number 6505 in Proceedings of SPIE, pp. 65050N-1 - 65050N-9, SPIE.

[6] W. M. Dietl and A. Uhl, "Robustness against unauthorized watermark removal attacks via key-dependent wavelet packet subband structures," in Proceedings of the IEEE International Conference on Multimedia and Expo, ICME '04, Taipei, Taiwan, June 2004.

[7] Houng-Jyh Wang and C.-C. Jay Kuo, "Watermark design for embedded wavelet image codec," in Proceedings of the SPIE's 43rd Annual Meeting, Applications of Digital Image Processing, San Diego, CA, USA, July 1998, vol. 3460, pp. 388-398. 